\title{
Adaptability of a Catalog Spacecraft Bus to Diverse Science Missions ${ }^{12} 3$
}

\author{
Edward A. Belte, Steven P. Thompson, Dominick Conte \\ Spectrum Astro, Inc. \\ 2214 Rock Hill Road, Suite \#101 \\ Herndon, VA 20170 \\ 703-742-7876 \\ John Jordan, Albert Lepore, Kendall Nii, David Olschansky, John Sutila \\ Spectrum Astro, Inc. \\ 1440 North Fiesta Blvd. \\ Gilbert, AZ 85233 \\ 480-892-8200 \\ www.spectrumastro.com \\ contact: ed.belte@specastro.com
}

\begin{abstract}
Over the past decade, the concept of using "offthe-shelf" Spacecraft (SC) buses for space science and earth science missions has become widespread. A "common bus" design approach has been used for Geosynchronous (GEO) communications satellites since the early 1970's. The success of using common bus designs for the manufacture of GEO communications satellites is due to the commonality of mission requirements and orbit geometry. Science missions, on the other hand, each have unique mission and instrument payload requirements that can vary widely, encompassing orbit geometry, instrument type and configuration, science target, SC attitude, operations concept, and launch scenario.
\end{abstract}

One of the most visible and successful implementations of "off-the-shelf" SC for science applications is the NASA Goddard Space Flight Center (GSFC) Rapid Spacecraft Development Office (RSDO) catalog, first released in 1997. In the current catalog (Rapid II), there are twenty-three different SC buses manufactured by eight aerospace companies.

This paper provides a case study describing the adaptation of Spectrum Astro's SA-200HP (High Performance) RSDO catalog SC bus to two very different Low Earth Orbiting (LEO) science missions, Coriolis and Swift, which were both procured via the RSDO.

Coriolis is a Department-of-Defense-sponsored sunsynchronous earth observation satellite whose primary instrument, WindSat, is designed to precisely measure the ocean surface wind vector.

Swift is a low inclination NASA Medium Explorer (MIDEX) mission to detect and characterize Gamma Ray
Bursts (GRBs). The Swift Observatory carries three separate telescopes.

In addition to describing how the catalog SC bus was applied to these missions, this paper discusses the unique features and benefits of the catalog bus approach to both the procuring agency and the industry bus provider. Misconceptions associated with the use of the catalog bus approach are also discussed.

\section{TABle of Contents}

1. INTRODUCTION. 1

2. SA-200HP RAPID II CATALOG BUS DESCRIPTION \& FLIGHT HERITAGE ...........3

3. AdAPTATION OF THE SA-200HP SC BUS TO THE CORIOLIS MISSION 7

4. ADAPTATION OF SA200-HP SC BUS TO SWIFT .10

5. SIMILARITIES AND DIFFERENCES BETWEEN CORIOLIS AND SWIFT ................................12

6. USE OF GSFC'S RSDO CATALOG ............14

7. ConCluSIONS .......................................15

8. REFERENCES ...........................................15

9. BIOGRAPHIES .......................................16

\section{INTRODUCTION}

Several U.S. companies have made significant investments developing "common" SC bus designs that can be used for a variety of SC mission profiles. The "core" designs are altered with hardware and software changes to adapt to each mission's requirements. In many instances, these common designs were developed from a "one-of-a-kind" SC manufactured for a single mission. The adaptation of a "one-

\footnotetext{
${ }^{1}$ 0-7803-7651-X/03/\$17.00 (C) 2003 IEEE

2 IEEEAC paper \#1226, Updated Dec 10, 2002

${ }^{3}$ Spectrum Astro Document \# 0000-EZ-U44352
} 
of-a-kind" SC into a flexible "core" design allows companies to save a significant amount of non-recurring engineering costs for future missions. Since the SC industry has become highly competitive in recent years, the savings are normally passed on to the customer.

A common bus design approach has been used for GEO communications satellites since the early 1970's. While companies do not stock GEO SC buses in their factories waiting for orders to materialize, they are able to quickly produce a SC bus for a given GEO mission with only minimal changes to their basic bus design, manufacturing, and integration procedures. The success of a common SC bus design for GEO communications satellites is due to the large and increasing number of missions at the GEO orbit, the commonality of mission requirements, and uniform orbit geometry (from mission to mission). Typically, the major variations in a GEO bus design involve only power levels and stability requirements.

Science missions, on the other hand, each have unique mission and instrument payload requirements that can vary widely, encompassing orbit geometry, instrument type and configuration, science target, SC attitude, operations concept, and launch scenario. A highly adaptable and flexible core SC bus design is necessary to respond effectively to the broad range of science requirements, while minimizing costs, fabrication schedules, and development risks. Several companies have developed such designs. The U.S. Government, as the largest procurer of science mission $\mathrm{SC}$, recognized the benefits of standardized designs. Their vision was to streamline the procurement process and schedule by compiling a list of "space qualified" core SC buses that could be purchased under a set of pre-specified contract terms. This approach has been tried previously by other agencies with mixed results. However, NASA Goddard's RSDO has been very successful, with seven SC awards in the past five years. The RSDO provides services to all space-faring federal agencies and their affiliates, as well as university-based principal investigators. They offer a catalog of SC buses that are available through an Indefinite Delivery Indefinite Quantity (IDIQ) contract.

Today's RSDO catalog [1] features eight aerospace companies offering twenty-three different SC buses. The catalog allows virtually any government agency or entity (corporation, university, etc.) to procure a SC utilizing RSDO resources for a nominal fee to cover expenses. In addition, the standard RSDO contract vehicle can be specifically tailored to meet the procuring organization's requirements. All catalog SC core bus entries must demonstrate flight qualification by showing direct heritage to a SC that, at minimum, has been integrated to a Launch Vehicle (LV). While the basic RSDO IDIQ contract has a period-of-performance of five years, the catalog has provisions for "on ramps" every six months, which allow vendors not currently in the catalog to propose core buses or vendors already in the catalog to add new core buses or update previously accepted core buses with technology improvements or new options. The initial RSDO catalog offering, Rapid I, was implemented in 1997. The Rapid II catalog, in effect since January 2000, introduced contracting with SC bus providers under Federal Acquisition Regulations (FAR) Part 12 acquisition. This allowed NASA to embrace commercial contract Terms and Conditions. A Rapid III catalog is planned to succeed Rapid II in 2004.

One of Spectrum Astro's RSDO catalog SC buses is the SA-200HP. The SA-200HP SC bus is a derivative of the Deep Space 1 (DS1) SC bus, designed and built for NASA's New Millennium Program (NMP). The bus development began with a Phase B Study in September 1995 and DS1 was launched three years later in October 1998 [2].

In early 1999, Spectrum Astro adapted its SA-200HP RSDO catalog bus to the Coriolis mission. The Coriolis SC was procured through RSDO by the United States Air Force (USAF), Space and Missiles Center Det. 12 (Space Test Program - STP) in Albuquerque, New Mexico. After a competitive award by Goddard's RSDO, the contract was transferred to the Air Force for administration [3]. Coriolis is a sun-synchronous earth observation satellite whose primary instrument, WindSat, is designed to precisely measure ocean surface wind vectors. WindSat is a 2-meter diameter, nadir-pointed microwave polarimetric radiometer [4]. The large radiometer antenna structure spins at 30 RPM, offering unique challenges in attitude stability and dynamics.

In the latter part of 1999, Spectrum Astro adapted the same SA200-HP RSDO catalog bus to the NASA Medium Explorer (MIDEX) Swift Mission. The Swift mission was procured through Goddard's RSDO and is managed by a Principal Investigator from GSFC [5]. Swift is a low inclination mission to detect and characterize GRBs [6] . The Swift Observatory carries three separate telescopes: an X-Ray Telescope (XRT), an Ultra-Violet Optical Telescope (UVOT) and a gamma-ray Burst Alert Telescope (BAT). A key feature of Swift is its ability to perform autonomous, rapid slews to point the high resolution, narrow field UVOT and XRT at GRBs detected by the BAT.

The Coriolis and Swift missions are excellent examples that demonstrate how a flexible core $\mathrm{SC}$ bus design can be tailored to meet varying mission requirements. The following sections describe how the SA-200HP SC bus was adapted to the Coriolis and Swift missions and also includes a discussion on the use of the RSDO catalog. 


\section{SA-200HP RAPID II CATALOG BUS DESCRIPTION \& FLIGHT HeRITAGE}

\section{SA-200HP Overview}

Spectrum Astro's SA-200HP SC bus is a scaleable, robust platform designed for a variety of space missions ranging from simple LEO operations to planetary missions demanding precision pointing and extended lifetimes. The core bus, illustrated in Figure 1, can be easily adapted to support LEO, Medium Earth Orbit (MEO), High Earth Orbit (HEO), GEO, and deep space applications on technology demonstration, earth science, and space science missions. The SC is capable of providing 3-axis control in a variety of modes that include inertial, solar, and nadir pointing, along with rotational and maneuver modes. A wide range of electric power and propulsion capabilities are also available. The SA-200HP is sized to support payloads using LV systems such as Taurus, Athena I-II, Titan II, Delta II and Atlas. Several upgrade options are available, including full redundancy, addition of GPS and magnetics (for reaction wheel momentum management), a deep space configuration, and ion propulsion. All of the bus subsystems used on the SA-200HP and its options are flight proven.

\section{SA-200HP Flight Heritage}

Spectrum Astro's SA-200HP SC bus draws its flight heritage from the successful DS1 mission. DS1 is a NASA NMP SC managed for NASA by the Jet Propulsion Laboratory (JPL) in Pasadena, California. The NMP is an advanced-technology development program NASA created to develop and flight qualify new technologies and mission concepts for use in future missions. The program is unique since it tests many of its advanced technologies in space flight rather than in a laboratory. By testing these new technologies in flight, NMP lowers the risk for future operational or science missions that are interested in using the technologies. The highly successful DS1, shown in Figure 2, was the first NMP mission.

The DS1 mission had two major objectives:

(1) Carry and validate 12 advanced technology payloads (some of which performed as primary SC subsystems).

(2) Conduct a comet flyby and return images and spectra to the earth for analysis.

Of the 12 technology payloads, the primary advanced technologies to be validated through in-flight demonstrations were:

(1) Solar Electric Propulsion (SEP) as the primary method of propulsion.

(2) Solar concentrator array as the primary means of powering the SC and its SEP subsystem.

(3) Autonomous on-board optical navigation as the primary means of navigating the SC.

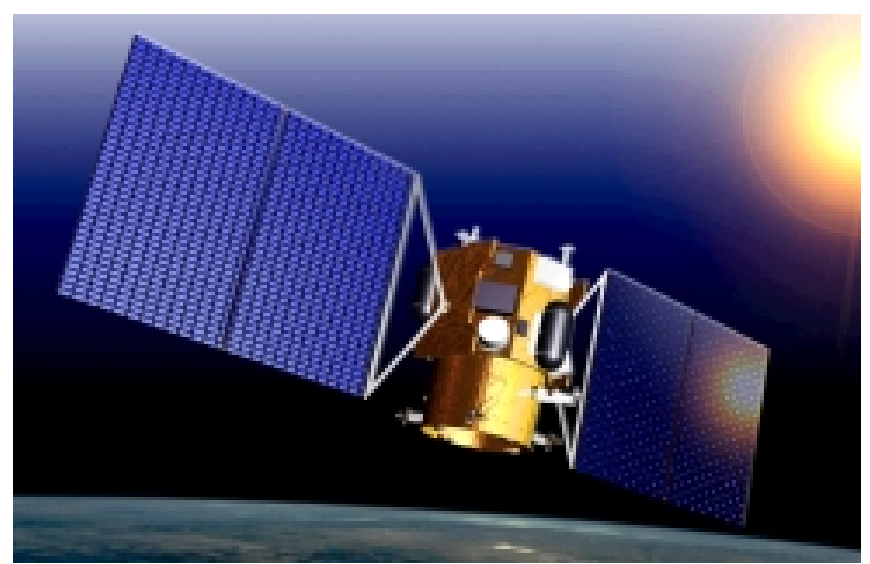

Figure 1 - Spectrum Astro's RSDO Catalog SA-200HP SC Bus in LEO

(4) Autonomous Remote Agent which is an artificial intelligence system that autonomously plans and executes spacecraft activities

(5) Miniature Integrated Camera Spectrometer (MICAS) to image the asteroid Braille and during the extended mission, comet Borrelly.

Spectrum Astro and JPL collaborated on the design and development of DS1. Spectrum Astro designed, manufactured, and integrated the $\mathrm{SC}$ bus and Electrical Ground Support Equipment (EGSE) used throughout SC and satellite Integration and Test (I\&T), while JPL had prime responsibility for overall systems engineering, development of the science and technology elements, system-level I\&T, launch, and mission operations.

Challenges met by Spectrum Astro on the DS1 mission included development of a High Voltage Power Converter

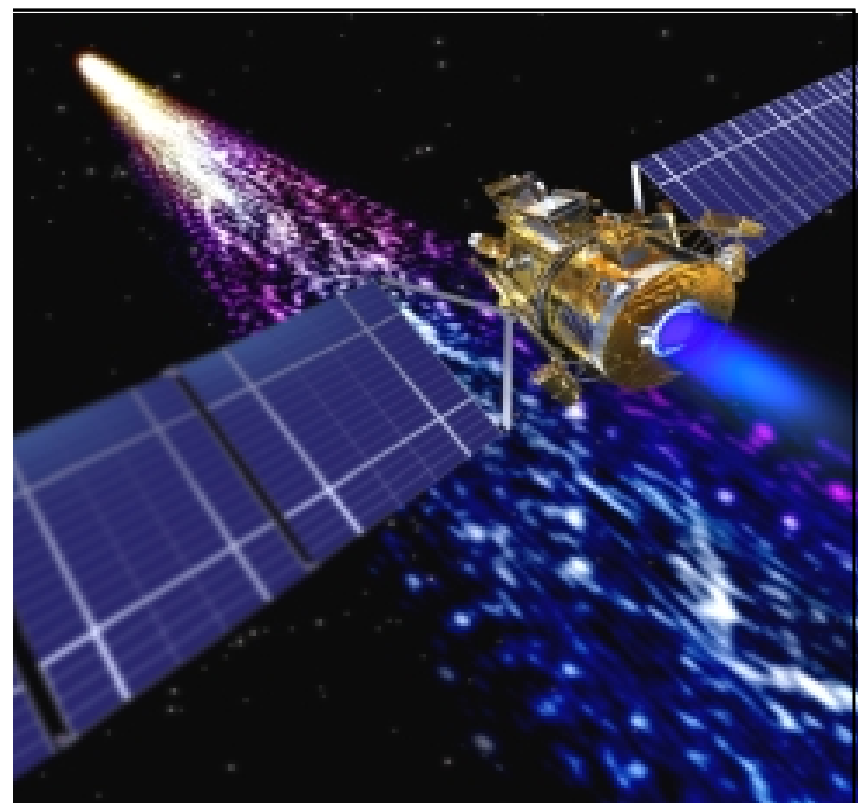

Figure 2 - Artist's Concept of Spectrum Astro's DS1 SC in Pursuit of Comet Borrelly. DS1 Validated a High Voltage SEP Subsystem as the Primary Method of Propulsion 
Unit (HVPCU) that efficiently transfers the nominal 100 VDC array output to the ion engine, as well as downconverting power to $28 \mathrm{VDC}$ for bus component and battery usage, and development of the data handling electronics for the $4 \mathrm{Mbps}$ MICAS instrument. Also, under a separate contract to Hughes (now Boeing), Spectrum Astro designed and developed the digital controller for the xenon ion engine.

The DS1 SC was launched on 24 October 1998 on a Delta 7326-9.5 Med-Lite LV from Cape Canaveral Air Station, Florida. The SC was placed into a heliocentric orbit. In July 1999, DS1 completed a flyby of the asteroid Braille at a closest approach distance of $15 \mathrm{~km}$. The primary mission was successfully concluded in September 1999. At that time, the mission was extended [7] to allow a rendezvous with the comet Borrelly [8]. In November 2000, DS1 reached its maximum geocentric range of $2.36 \mathrm{AU}$. The comet Borrelly rendezvous occurred in September 2001 at $1.36 \mathrm{AU}$ from the sun approximately eight days after the comet's perihelion [9]. In December 2001, after more than two years in heliocentric orbit, the DS1 mission was intentionally terminated having successfully completed its primary and extended missions and depleting all of its xenon fuel.

From the successful launch and performance of the DS1 SC, Spectrum Astro was able to utilize the basic DS1 SC bus design and offer it for consideration as a core bus in NASA's RSDO Rapid II SC catalog.

\section{SA-200HP Core System Description}

The SA-200HP's core capabilities and features provide all basic bus subsystem and instrument interface functions using highly reliable single-string elements combined with fully redundant Electrical Power Subsystem (EPS) and Command and Data Handling (C\&DH) subsystems. The second and third columns contained in Figure 13 (in Section 5) outline key performance parameters for the SA-200HP core SC bus including the full redundancy option. While the SC bus is capable of being launched on several LVs, the performance parameters contained in the table are referenced to a $600 \mathrm{~km}$ circular, $28.5^{\circ}$ orbit using a Taurus launch. The core bus mass is $354 \mathrm{~kg}$ (dry) and the structure has a payload mass capacity of up to $666 \mathrm{~kg}$. The core bus has a design lifetime of four years and is designed to be radiation tolerant to 25 krad (softest parts) which exceeds the majority of predicted worst case LEO conditions. Adding the Full Redundancy and GPS/Magnetics Options further enhances mission lifetime and increases the core bus mass to $374 \mathrm{~kg}$ (dry). The functional block diagram for the SA-200HP core bus with the Full Redundancy and GPS/Magnetics Options is depicted in Figure 3. The following paragraphs describe each of the major subsystems depicted in the functional block diagram.

Structures/Mechanisms and Thermal-The structural subsystem of the SA-200HP SC bus consists of a cylindrical aluminum launch adapter coupled to Spectrum Astro's patented "space frame" - a low part count aluminum frame that minimizes weight and provides a simple, low risk manufacturing approach. The frame consists of three primary components: longerons, stringers, and shear panels. Loads are directed through the longerons, which mate directly to the LV adapter. Variation of the load path is minimized, which stiffens the structure while reducing the required mass. The frame is closed out with aluminum honeycomb/aluminum facesheet shear panels. As shown in Figure 4, the bus structure consists of two modules, the lower LV adapter module contains the propulsion/reaction control subsystem which enables complete buildup of the propulsion subsystem in parallel with the SC bus upper module which contains all the SC bus electronics. SC bus components are typically mounted on the exterior of the upper module allowing for ease of access during I\&T operations. The thermal control subsystem is cold-biased with radiators and Multi-Layer Insulation (MLI) blankets to minimize fluctuations in temperature. Thermostaticallycontrolled heaters are used to keep components at desired temperatures. Payloads can be either thermally-isolated or thermally-coupled to the bus, depending on their individual needs.

Electrical Power-The SA-200HP has two solar array wings located on opposite sides of the SC. Each two-panel wing has biaxial articulation to maximize power generation regardless of SC attitude or orbit. The array generates just over $2000 \mathrm{~W}$ at End-of-Life (EOL). In a typical LEO, the core bus power subsystem can support payload power levels of up to $650 \mathrm{~W}$ Orbit Average Power (OAP) and a worstcase bus power at $354 \mathrm{~W}$ OAP. With the Full Redundancy and GPS/Magnetics Options the payload power levels are up to $639 \mathrm{~W}$ OAP with a worst-case bus power at $367 \mathrm{~W}$ OAP. The array is populated with triple-junction Gallium Arsenide (GaAs) cells. Two 50-Ahr $\mathrm{NiH}_{2}$ Single Pressure Vessel (SPV) batteries provide power during eclipses, launch, or in the event of an anomaly that causes the SC to lose attitude. The bus voltage is maintained at $28+/-6 \mathrm{~V}$ and is supplied to both the SC bus and payload by an internally redundant Power Distribution Unit (PDU). Redundant Charge Control Units (CCUs) charge each battery using both software and hardware charging profiles. The power system provides autonomous shedding of payload and non-essential loads to safe the vehicle in an anomaly.

Telecom-Command and telemetry are via an S-band link using a Space Tracking and Data Network (STDN) S-band ranging transponder. Uplink is at $2 \mathrm{kbps}$; either stored or real-time telemetry can be downlinked at $128 \mathrm{kbps}$. Hemispherical pattern S-band receive and transmit antennas are located on opposite faces of the SC to provide nearomnidirectional coverage. The receive portion of the transponder is always powered and ready to receive and accept commands. While the X-band transmitter is capable of rates of up to $320 \mathrm{Mbps}$, in the SA-200HP configuration 


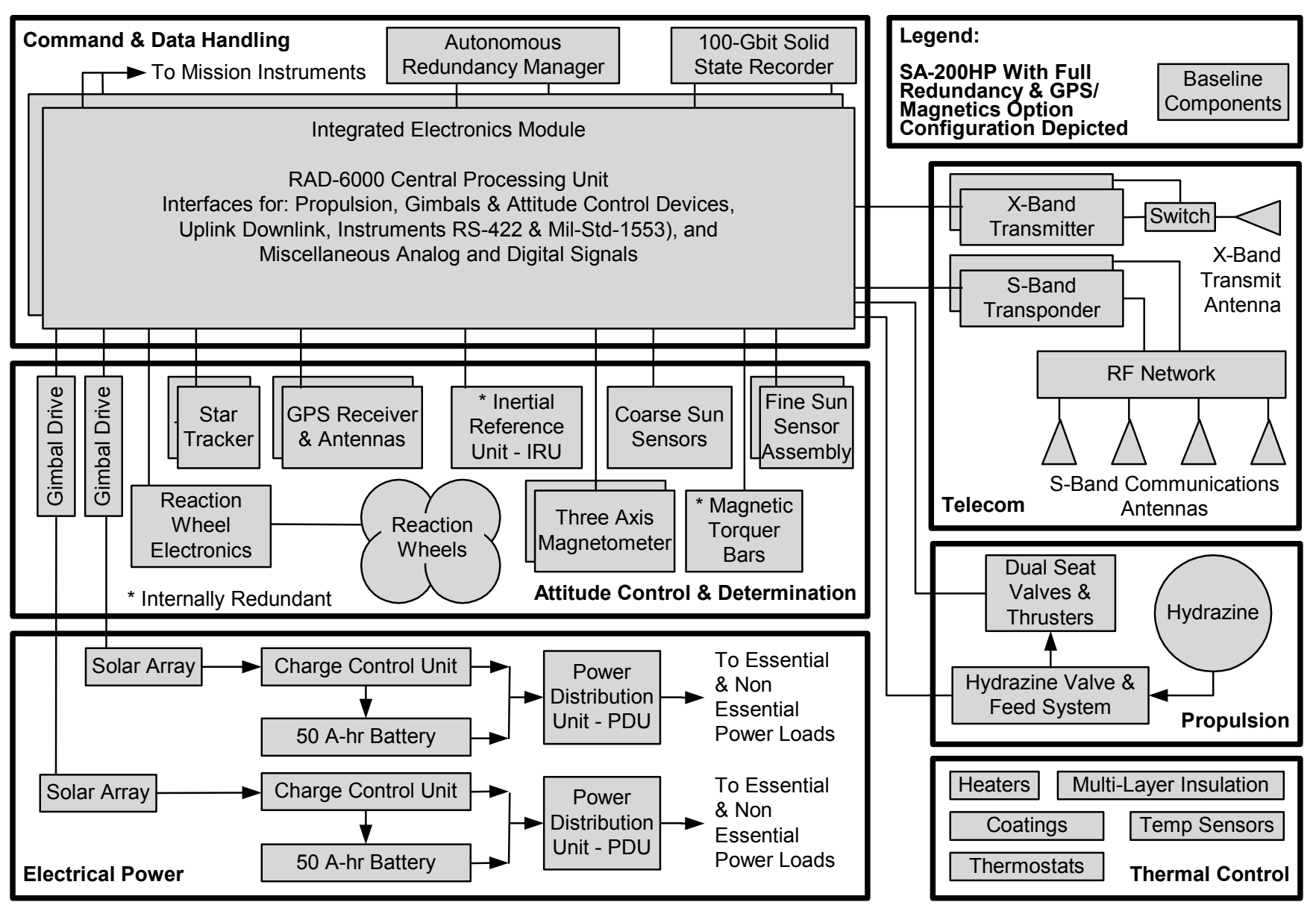

Figure 3 - SA-200HP Block Diagram with Full Redundancy and GPS/Magnetics Options

with a fixed, medium-gain antenna, return payload science data rates up to $80 \mathrm{Mbps}$ can be supported. A $2.5 \mathrm{Mbps} \mathrm{S}-$ band science data downlink is also available using the STDN S-band transponder. All communications links are Consultative Committee for Space Data Systems (CCSDS) compatible for communication with NASA STDN ground terminals.

$C \& D H$ - The C\&DH subsystem utilizes a BAE Systems RAD6000-based Central Processing Unit (CPU) board operating at $20 \mathrm{MHz}$, providing over $17 \mathrm{MIPS}$ of processing performance and 128 Mbytes of command, telemetry, and data storage. A Versa-Module Eurocard (VME) backplane architecture allows for flexibility and standardized interfaces. The flexible and flight-proven C\&DH consists of two block-redundant VME "sides" that are contained within a single chassis. An Autonomous Redundancy Management (ARM) board monitors performance and, via watchdog timers, can cause automatic fail-over to the redundant side. The Integrated Electronics Module (IEM) contains a series of Printed Circuit Boards (PCBs) that interface, control, and monitor all of the $\mathrm{SC}$ and payload electronic equipment. The IEM also contains an Internal Power Converter Unit (IPCU) board that converts the nominal $28 \mathrm{~V}$ bus voltage to +5 and $+/-15 \mathrm{~V}$ power for the processor and other cards in the IEM, and selected Attitude Control Subsystem

(ACS) components.

Payload data storage is provided by a 100 -Gbit Solid State Recorder (SSR). Flight software was developed using VxWorks and $\mathrm{C} / \mathrm{C}++$ and has flight heritage on the DS1, Miniature Sensor Technology Integration (MSTI) -3, MightySat II.1, and Reuven-Ramaty High Energy Solar Spectroscopic Imager (RHESSI) missions.

Attitude Control - Standard operating modes include nadir pointing, 3-axis slew and point, solar inertial, inertial (stellar/Inertial Measurement Unit (IMU) propagated), and surface point-tracking with full maneuver capability. Four reaction wheels are used (three are required) to provide three-axis, zero momentum bias ACS. An internallyredundant IMU senses attitude rates, while the four reaction wheels manage and store momentum. Momentum unloading is performed by thrusters or by magnetic torquer bars (with the GPS/Magnetics Option). A high precision star tracker provides attitude knowledge. Coarse sun sensors are utilized as attitude reference devices during safe hold mode. All the attitude control sensors and actuators consist of unmodified, previously flown equipment. System performance in the reference orbit is $+/-18$ arcsec control and $+/-0.5$ arcsec knowledge (with on-orbit instrument to optical bench 
calibration). The high accuracy attitude knowledge capability can support the vast majority of science missions. $\mathrm{SC}$ jitter for the bus alone has an amplitude of less than 10 arcsec. While ephemeris knowledge (better than $5-\mathrm{km}$ accuracy) can be provided using standard STDN S-band coherent ranging, the optional Global Positioning System (GPS) receiver can provide 3-axis ephemeris knowledge of 90m (3 sigma) or better.

Propulsion/Reaction Control-The SA-200HP has a simple monopropellant hydrazine blowdown propulsion system that can be used for both orbit transfer and attitude control. The propulsion system uses four 5-N (one pound) thrusters located on the aft end of the SC aligned parallel to the vehicle centerline, and four $1-\mathrm{N}(0.2$ pound $)$ thrusters oriented perpendicular to the centerline for attitude control. The 56-cm diameter spherical titanium tank has a maximum hydrazine capacity of $67 \mathrm{~kg}$.

\section{SA-200HP Options}

The SA-200HP core SC bus is available with several standard and flight proven options. The most utilized options are the Full Redundancy and GPS/Magnetics options. Our experience has shown that the majority of longlife LEO missions employ this option. For this reason, we have included it in Figures $3 \& 4$ and have used it in establishing a SC "baseline" for many of the more "demanding" LEO missions such as Coriolis and Swift. The following sections describe some of the standard RSDO catalog options that are available for the SA-200HP core SC bus.
Full Redundancy - This option offers improved mission lifetime and performance for LEO missions that extend over an on-orbit operating period of four years. While the SA$200 \mathrm{HP}$ baseline already has fully redundant C\&DH and EPS subsystems, this option significantly increases the probability of mission success by incorporating full redundancy for the remaining Attitude Control components and Telecom components.

GPS/Magnetics - A redundant GPS navigation system is added improving onboard ephemeris accuracy to $90 \mathrm{~m}$, a feature that is critical to earth-referenced observations and measurements or missions requiring precise relative SC positions. The addition of the magnetic-based attitude control (consisting of a three-axis magnetometer and three orthogonal magnetic torquer bars) provides wheel momentum management without the use of propellant (a life-limiting item).

Deep Space Configuration-The Deep Space option provides a NASA Deep Space Network (DSN) compatible deep space exploration and interplanetary configuration. The primary change includes elimination of the (LEO) $\mathrm{S} \& \mathrm{X}$ band equipment and replacement with a Small Deep Space Transponder (SDST), elimination of ACS hardware that is not applicable to deep space missions, downsizing of the battery (since there are few eclipses), and downsizing of the SSR.

Electric Ion Propulsion-This option adds the DS1 xenon Ion Propulsion System (IPS) to the core SA-200HP configuration. It provides a highly efficient (up to $3280 \mathrm{sec}$

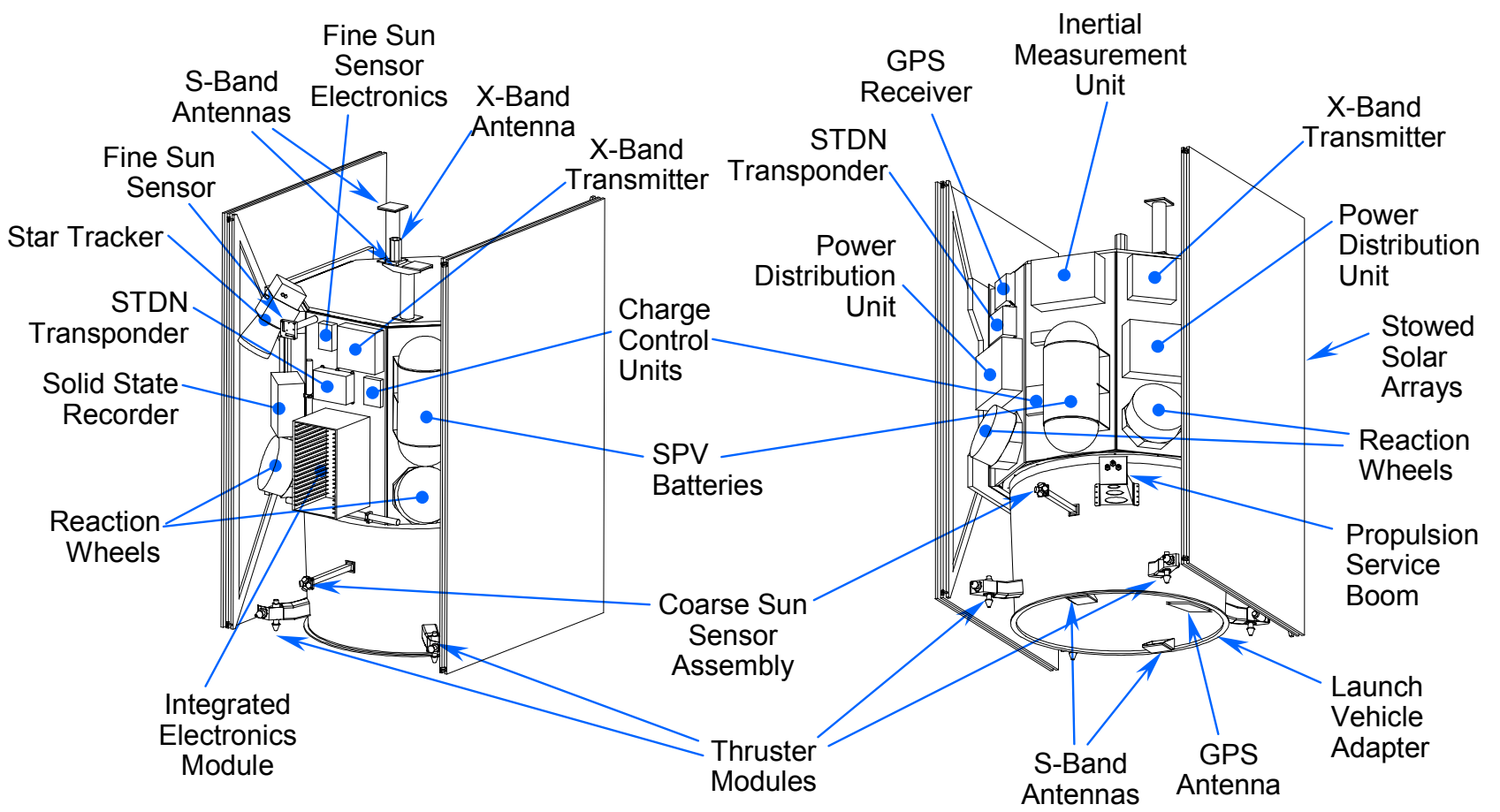

Figure 4 - Spectrum Astro's SA-200HP Core SC Bus Equipment Layout with Full Redundancy and GPS/Magnetics Options 
Isp), low thrust propulsion capability that dramatically increases propellant efficiency, enabling accommodation of high $\Delta \mathrm{V}$ missions on smaller LVs that would be unachievable using chemical propulsion. The resulting configuration is very similar to the flight DS1 configuration. Combining the previous Deep Space and Ion Engine options converts the SA-200HP core SC bus to the flight-proven DS1 configuration, providing a high $\Delta \mathrm{V}$ capability suitable for deep space and interplanetary missions.

\section{AdAPTATION OF THE SA-200HP SC Bus TO THE CORIOLIS MISSION}

\section{Mission Description}

Coriolis is a USAF STP demonstration and risk reduction mission. It has a three-year mission life (five-year goal), with primary objectives of demonstrating remote sensing of the ocean surface wind vector from space, collecting a comprehensive global data set, and transmission of real-time data products directly to field users. This effort is being undertaken as an essential risk reduction precursor for the National Polar-Orbiting Operational Environmental Satellite System (NPOESS). A secondary mission objective is to monitor solar activity with the goal of more accurately predicting geomagnetic disturbances to orbiting satellites. Spectrum Astro is responsible for the development, manufacture, integration, and verification of the SC bus; payload-to-SC integration; space vehicle-to-LV integration; interfacing with the Air Force Satellite Control Network (AFSCN) and commercial X-band ground systems; provision of the launch site payload processing facility; and early orbit checkout. Coriolis was procured by the USAF through NASA RSDO, representing the first outside agency use of the catalog bus concept. After the procurement, NASA transferred administrative management of the contract to the Air Force. Coriolis is to be launched in December 2002 into a parking orbit of $275 \times 808 \mathrm{~km}$ by an Air Force Titan II LV and utilized its own onboard propulsion system to achieve the final $830-\mathrm{km}$ circular, sunsynchronous, twilight orbit.

Coriolis, illustrated in Figures 5 and 6, carries two science payloads: a passive radiometer (known as WindSat) and three solar imaging cameras (known as Solar Mass Ejection Imager (SMEI)). WindSat is a Navy polarimetric microwave radiometer experiment to passively measure the ocean surface wind vectors from space. The payload has a mass of $341 \mathrm{~kg}$, uses an average of $434 \mathrm{~W}$ per orbit, and stands about $3.6 \mathrm{~m}$ tall. It includes a $125 \mathrm{-kg}$ rotating antenna platform that spins about the earth pointing yaw axis at 29.6 RPM, providing a 45-deg scan Field Of View (FOV) for its reflector. Collected radiation is focused onto an array of feedhorns, processed, and relayed to the SC for storage and downlink. The USAF Research Laboratory's SMEI payload monitors solar activity through three separate cameras with a collective 3 x 180o FOV, allowing full sky coverage during each orbit. A SMEI Data Handling Unit (DHU) processes the camera data and transfers it to the SC for storage and downlink. The SMEI payload has a mass of $33 \mathrm{~kg}$ and draws up to $83 \mathrm{~W}$ per orbit. The entire SC measures approximately 2.3-meters in diameter by 4.7-meters high (stowed). In the deployed configuration with the solar arrays extended, the overall length becomes 7.1 meters. The total weight at launch was $775 \mathrm{~kg}$ including $82 \mathrm{~kg}$ of hydrazine propellant (excluding the LV adapter). While the overall configuration of the Coriolis SC may appear quite different from the SA-200HP, structurally, the Coriolis SC bus retains the modularity concept. Coriolis features three modules, a propulsion module (connected to the propulsion service boom in Figure 6), a SC bus electronics module with externally mounted SC bus components (and some payload items such as the SMEI cameras), and the WindSat radiometer payload. Coriolis is 3-axis stabilized with pointing knowledge of $0.02^{\circ}$ and control to $0.2^{\circ}$. The WindSat payload provides its own spin momentum cancellation with a large momentum wheel.

Key technical challenges met on the Coriolis Program include the requirement for a SC bus with extremely lowlevel Electromagnetic Interference (EMI) to preclude interference with WindSat's passive microwave receiver; physical accommodation of WindSat's large size and mass; and a robust safing architecture for the large spinning assembly.

Coriolis [10] is a near fully redundant, configure-to-order adaptation of the SA-200HP catalog bus, accommodating a

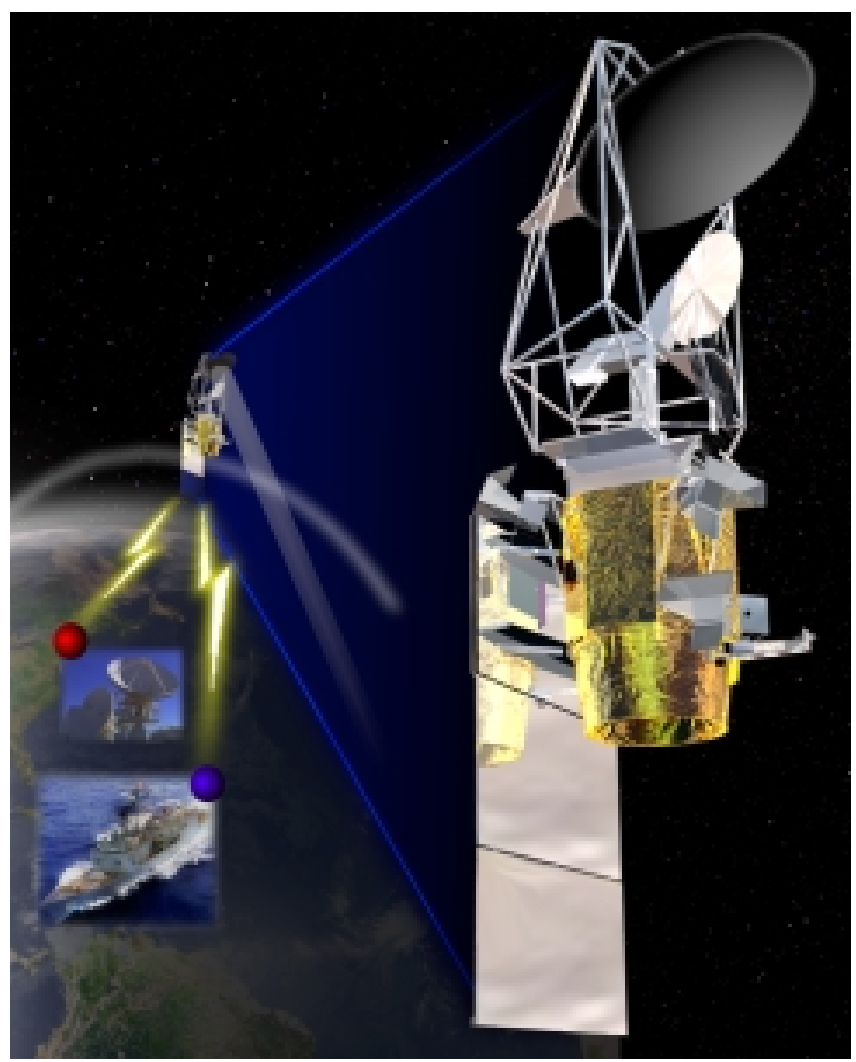

Figure 5 - Artist's Concept of the Coriolis SC 


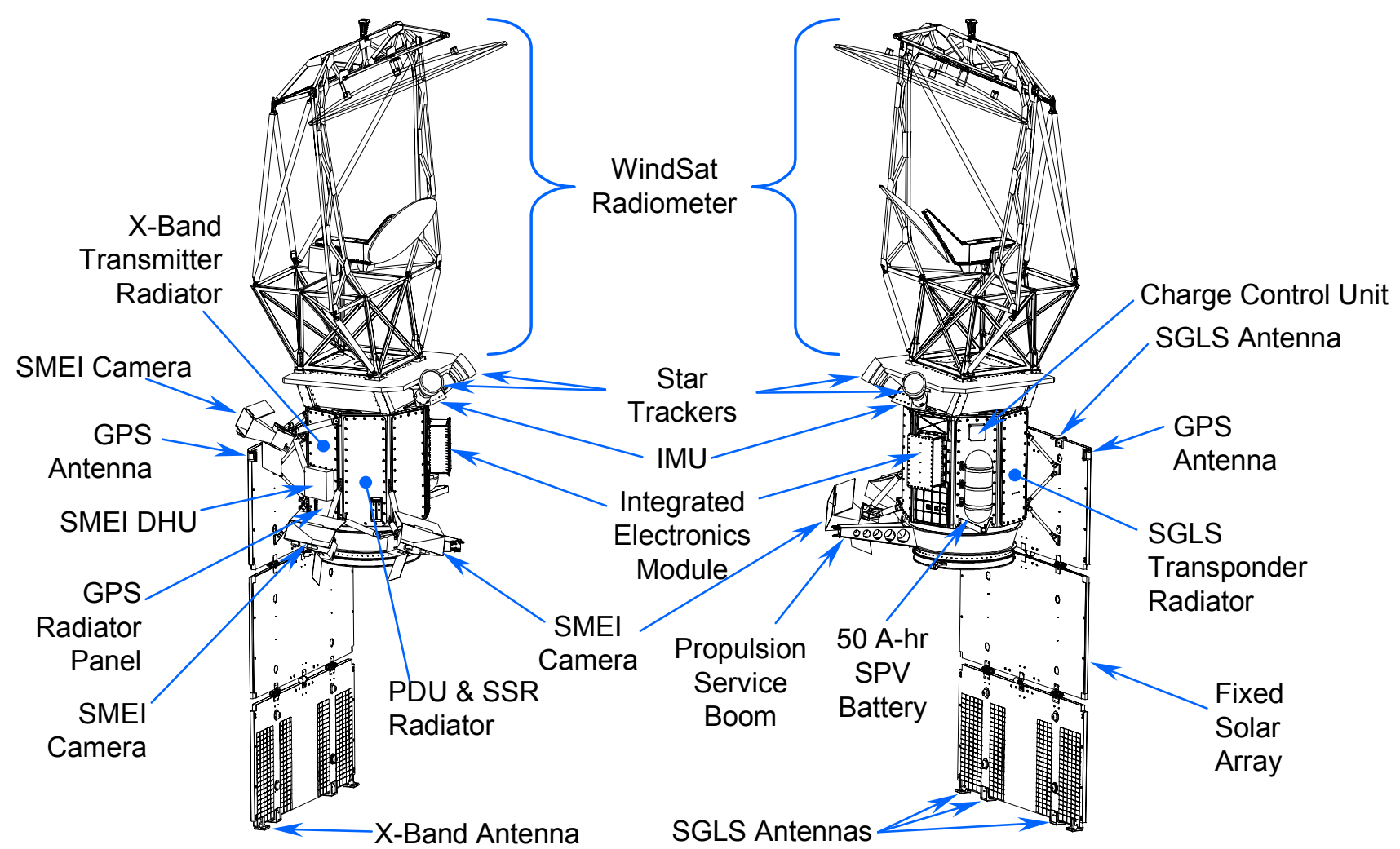

Figure 6 - Coriolis SC Configuration

large, unique instrument suite. It consists of the SA-200HP core catalog bus coupled with several mission unique modifications. The following paragraphs give a brief description of each of the Coriolis SC bus subsystems. The functional block diagram for the Coriolis SC bus is depicted in Figure 7. The following paragraphs describe each of the major subsystems. A description of the subsystem level changes to the SA-200HP SC bus that were necessary to accommodate the Coriolis mission, is tabulated in Figure 8.

$C \& D H$ - With just minor differences in electronics card configurations, the Coriolis SC bus uses the same IEM as the SA200-HP centered around the BAE RAD6000 CPU with two block-redundant VME bus "sides". The ARM board monitors system performance and, via watchdog timers, can cause automatic fail-over to the redundant side. The SA-200HP GDE boards have been eliminated since the solar arrays on the Coriolis mission are fixed deployable panels instead of the dual axis articulated arrays used on the SA-200HP. The size of the SSR has been reduced from 100 Gbits to 34 Gbits since the science data volume is considerably less than 100 Gbits. This change is essentially a reduction of the number of memory boards within the SSR. Redundant 1750A-based Intelligent Bus Controller boards have also been added to provide the capability to perform pre-processing of payload data prior to storage. This board also contains the 1553 interface to support the payloads. An Auxiliary Ground Support Equipment (AGSE) board interfaces the SC to Ground Support Equipment (GSE) to facilitate ground testing.

Attitude Control-The Coriolis SC utilizes a three-axis ACS. Four reaction wheels provide a pitch momentum bias and a stable nadir-pointing platform for the large spinning WindSat payload. A three-axis magnetometer senses the earth's magnetic field orientation, while three magnetic torquer bars unload excess momentum. Attitude knowledge is provided by redundant star trackers. Coarse sun sensors are utilized as attitude reference devices during safe hold mode. Redundant IMUs measure SC inertial rates and linear acceleration to assist in closed loop attitude and propulsion maneuvers. GPS is used for precision position and velocity knowledge to better than $100 \mathrm{~m}$ and $10 \mathrm{~cm} / \mathrm{sec}(\mathrm{rms})$ about all axes. System performance is 324 arcsec control and 194 arcsec knowledge ( $3 \sigma$, steady-state WindSat spin). SC jitter is less than $108 \operatorname{arcsec}(3$-axis, $3 \sigma)$.

Electrical Power-Coriolis utilizes a single three-panel solar array that deploys along the nadir direction and remains fixed thereafter. The array produces $1174 \mathrm{~W}$ under worst-case EOL conditions (i.e., after three years on orbit). The array is populated with dual junction GaAs cells. A 50Ahr $\mathrm{NiH}_{2}$ SPV battery is used to provide power during eclipses, launch, or if SC attitude is lost. The solar array also has a few strings of cells on the "backside" to power the essential SC bus components in the event of attitude loss. The power bus voltage is maintained at $28+/-6 \mathrm{~V}$ and is 


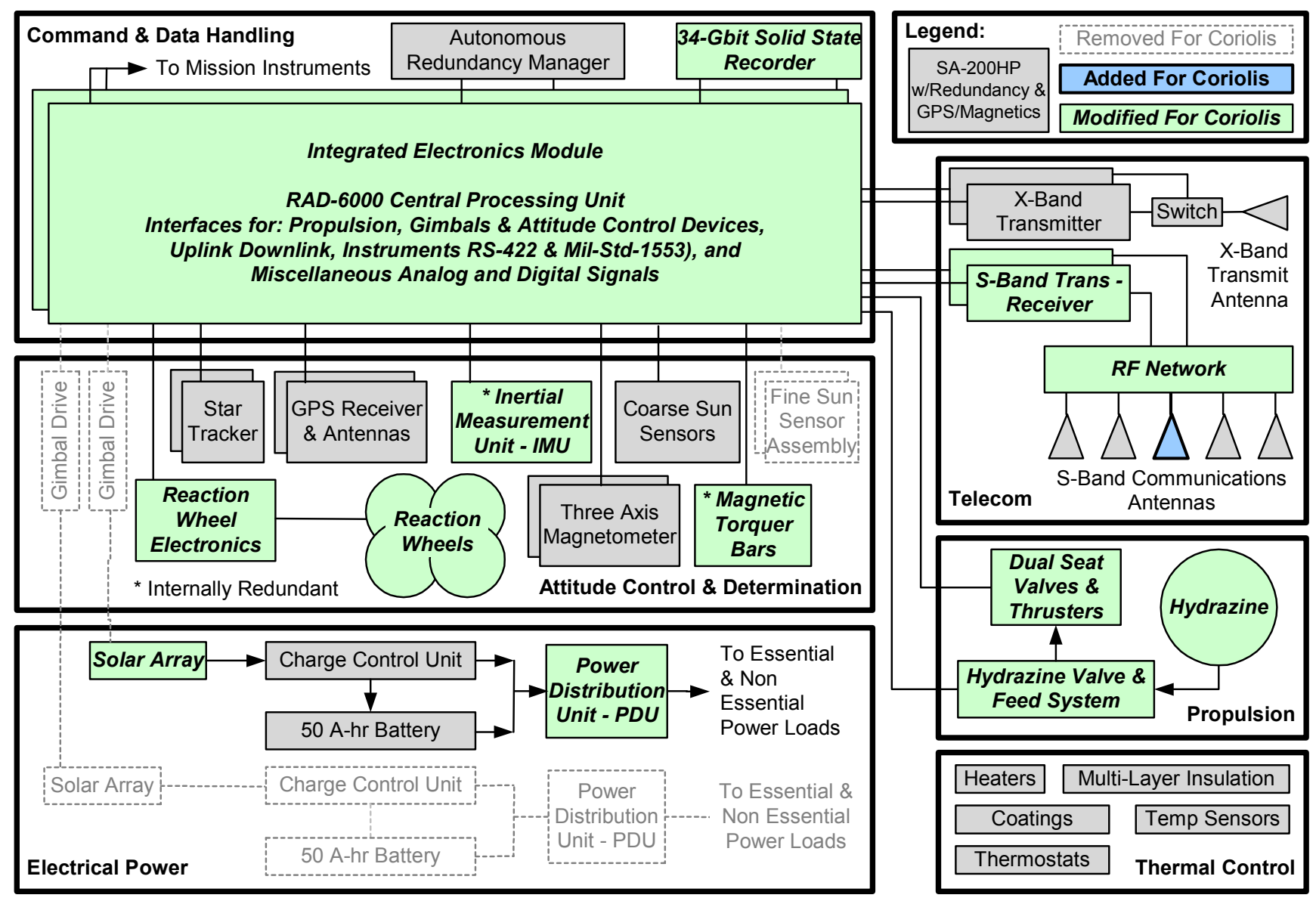

Figure 7 - Coriolis Block Diagram Showing Revisions, Additions and Deletions to the SA-200HP Baseline SC Bus Design

distributed to SC bus components and payload by the PDU. A CCU charges the battery using both software and hardware charging profiles. An IPCU provides +5 and $+/-$ $15 \mathrm{~V}$ to the various SC subsystem components requiring lower voltages. The power system provides autonomous load shedding for payload and non-essential loads to safe the vehicle in the event of an anomaly.

Telecom-Commanding and telemetry transfer are performed over an S-band link using redundant Space Ground Link System (SGLS) S-band ranging transponders with embedded encryption. As with the core bus, the uplink is at $2 \mathrm{kbps}$; either stored or real-time telemetry can be downlinked at $128 \mathrm{kbps}$. Hemispherical pattern S-band receive and transmit antennas are located on both the nadir and zenith faces of the SC to provide near-omnidirectional coverage in the event of an anomaly. The receive portion of both transponders is always powered enabling both transponders to receive and accept commands at all times. Payload science data is returned via X-band at a selectable data rate of either 25.6 or $51.2 \mathrm{Mbps}$ by redundant transmitters and a fixed, nadir facing, medium gain antenna. The X-band data stream is CCSDS compatible. There is also a real-time $256 \mathrm{kbps}$ S-band science data stream that can be broadcast directly to small portable ground terminals for tactical and/or science users in the field. This $256 \mathrm{kbps}$ data stream is transmitted using the backup S-band transponder.

Propulsion-Coriolis has a simple, monopropellant blowdown propulsion system that is used to correct LV insertion errors and raise the vehicle from its elliptical parking orbit to a circular operational orbit. No further stationkeeping maneuvers are planned after the operational orbit has been achieved (the orbit will be allowed to drift slightly over time). The system provides a total delta- $\mathrm{V}$ capability of $228 \mathrm{~m} / \mathrm{s}$. The propulsion system uses four $5-\mathrm{N}$ (one-pound) thrusters located on the aft end of the SC, each aligned parallel to the vehicle centerline. The thruster Isp ranges between 217 and 229 seconds depending on inlet pressure. The $59-\mathrm{cm}$ diameter titanium tank has a capacity of $90 \mathrm{~kg}$ of hydrazine.

Structures/Mechanisms and Thermal Control-The Coriolis structure is fabricated from aluminum honeycomb/aluminum facesheet panels. The bus module functions as a sealed Faraday cage to reduce component emissions to low levels preventing interference with the highly sensitive WindSat payload. There is a four-point bolted mount for the WindSat payload and the ACS Optical Bench is connected via flexures directly to WindSat minimizing structural misalignment. The three-panel, non-articulating solar array 


\begin{tabular}{|c|c|c|}
\hline Subsys & Modification & Rationale \\
\hline \multirow[t]{2}{*}{ C\&DH } & $\begin{array}{l}\text { Reduce size of SSR from } 100 \text { Gbits to } 34 \\
\text { Gbits }\end{array}$ & Additional memory unnecessary to meet mission requirements \\
\hline & Eliminate gimbal interface & No gimbals needed for Coriolis since solar array is fixed \\
\hline \multirow{3}{*}{$\begin{array}{l}\text { Attitude } \\
\text { Determination } \\
\text { and Control } \\
\text { Subsystem } \\
\text { (ADCS) }\end{array}$} & $\begin{array}{l}\text { Replace high accuracy Inertial Reference } \\
\text { Unit (IRU) with moderate accuracy IRU }\end{array}$ & IRU selection dictated by mission requirements \\
\hline & Delete fine sun sensors & Not necessary for mission operation \\
\hline & $\begin{array}{l}\text { Change wheel configuration for } \\
\text { operation in Pitch Momentum Bias } \\
\text { (PMB) mode }\end{array}$ & Pitch momentum bias adequate for mission \\
\hline \multirow[t]{2}{*}{$\begin{array}{l}\text { Electrical } \\
\text { Power }\end{array}$} & $\begin{array}{l}\text { Delete dual axis gimbaled solar array; } \\
\text { replace with single three panel } \\
\text { deployable stationary solar array }\end{array}$ & $\begin{array}{l}\text { Due to unique orbit (twilight sun sync), Coriolis array can be a } \\
\text { fixed flat panel }\end{array}$ \\
\hline & Delete one 50-Ahr SPV battery & $\begin{array}{l}\text { Redundant batteries not required to meet Coriolis mission } \\
\text { reliability requirements }\end{array}$ \\
\hline \multirow[t]{3}{*}{ Telecom } & $\begin{array}{l}\text { Replace S-band STDN transponder with } \\
\text { SGLS transponder with embedded } \\
\text { encryption/decryption }\end{array}$ & USAF missions require SGLS with encryption \\
\hline & Add 256 kbps tactical downlink & Mission requirement \\
\hline & $\begin{array}{l}\text { Larger beamwidth X-band antenna with } \\
\text { increased power on X-band transmitter }\end{array}$ & Necessary to provide full earth coverage \\
\hline Propulsion & $\begin{array}{l}\text { Increase tank size; delete four ACS } \\
\text { thrusters }\end{array}$ & $\begin{array}{l}\text { Additional propellant required for Coriolis ( } 82 \mathrm{~kg} \text { vs. } 67 \mathrm{~kg} \text { of } \\
\text { catalog vehicle); four small ACS thrusters, used for attitude } \\
\text { adjustment, not required }\end{array}$ \\
\hline Structure & $\begin{array}{l}\text { Modify SA-200HP structure to } \\
\text { accommodate Coriolis mission } \\
\text { requirements (EMI Faraday cage) }\end{array}$ & WindSat Payload EMC/EMI Mission requirement \\
\hline
\end{tabular}

Figure 8 - Subsystem Summary of Coriolis Mission Unique Changes to the SA-200HP SC Bus

uses a simple, reliable two-step deployment sequence. The thermal control subsystem is cold-biased with radiators and MLI blankets to minimize fluctuations in temperature. Thermostatically controlled heaters are used to keep components at desired temperatures. The WindSat interface is thermally isolated, but the SMEI cameras are thermally coupled to the bus.

\section{AdAPTATION OF SA200-HP SC BUS TO SWIFT}

\section{Mission Description}

Swift is a NASA MIDEX mission whose objective is the study of GRBs. The Swift Observatory detects GRB events and autonomously performs rapid slews to view the event with multi-spectral instruments that accurately locate the burst and perform scientific analysis. An artist's concept of Swift is presented in Figure 9.

GSFC's RSDO competitively selected Spectrum Astro as their teammate to supply the SC bus, perform payload to SC integration, perform space vehicle-to-LV integration, and early orbit checkout. Launch is scheduled for September 2003 aboard a Delta 7420 LV from Kennedy Space Center (KSC). Swift's mission life is a minimum of two years with a five-year goal. The primary design challenge on the Swift mission is to autonomously and rapidly slew and settle this large observatory, enabling detailed GRB observations as soon as possible after detection. Slews of up to $50^{\circ}$ must be completed in less than 75 seconds, while slews between $50^{\circ}$ and $180^{\circ}$ must be completed within 270 seconds. Additionally, these slews require onboard autonomy for predicting and planning each slew, while satisfying multiple pointing constraints for instrument safeguarding (i.e., avoid

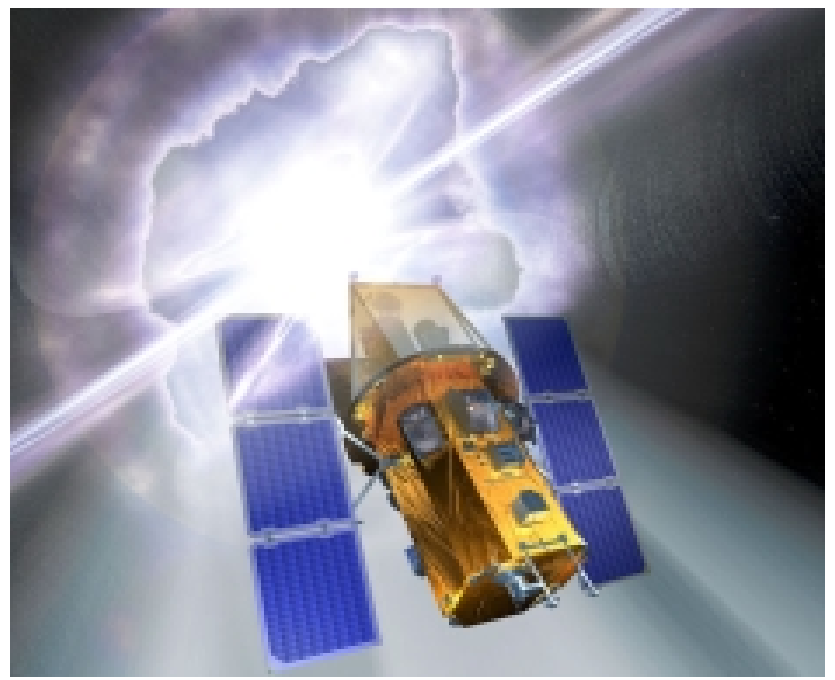

Figure 9- Swift's Mission is to Detect GRBs and Record Their $\gamma$-ray, x-ray, UV, and Optical Emissions 
pointing the instrument apertures at the earth, sun, moon, or along the SC velocity direction). Once slewing is complete, pointing knowledge must be maintained within 3 arcseconds in pitch and yaw, and within 1.5 arcminutes in roll. The pointing control error must be less than 3 arcminutes in pitch and yaw, and less than 4.5 arcminutes in roll. Pointing stability must be 1 arcsecond over 10 seconds in pitch and yaw, and 1.2 arcminutes over 1,000 seconds in roll.

The Swift SC illustrated in Figure 10 measures 1.8 meters in diameter by 5.6 meters high (stowed). In the deployed configuration with the solar arrays extended, the overall width is increased to 5.4 meters. The total weight at time of launch is $1,455 \mathrm{~kg}$. The SC communicates via S-band. The $\mathrm{C} \& \mathrm{DH}$ architecture is very similar to the SA-200HP catalog bus as it shares the same core software infrastructure and uses the same 1553 data bus controllers. The direct energy transfer EPS architectures are essentially identical, utilizing large deployable solar array wings, similar CCUs, and $\mathrm{NiH}_{2}$ batteries. The EPS services instrument loads of $695 \mathrm{~W}$ OAP. Both telecommunications subsystems utilize S-band and real-time Tracking and Data Relay Satellite System (TDRSS) links, including CCSDS protocols, and Reed Solomon encoding. Similar Zero Momentum Bias (ZMB) three-axis stabilized attitude control, star tracker-based attitude determination, and onboard autonomy and fault management are utilized. Swift utilizes a standard Delta II LV interface.

Swift [11], like Coriolis, is a redundant, configure-to-order adaptation of the SA-200HP catalog bus, fully accommodating a large instrument suite. The following paragraphs give a brief description of each of the Swift SC bus subsystems. The Swift SC functional block diagram is shown in Figure 11. A description of the subsystem level changes to the SA-200HP SC bus that were necessary to accommodate the Swift mission is given in Figure 12.

$C \& D H$ - The Swift SC bus uses the same fully block redundant IEM as the SA-200HP core vehicle with minor differences in electronics card configurations. The size of the SSR has been reduced from 100 Gbits to 32 Gbits. A Bus Controller Interface Board has been added to provide 1553 interfaces handling of payload data. A General Purpose Board (GPB) has also been added to provide additional channels for monitoring bi-levels, temperatures, voltages and currents. It also provides LV interfaces and an interface to GSE.

Attitude Control-The Swift SC is in a low inclination circular orbit and utilizes a three-axis stabilized ACS. Attitude knowledge is provided by set of redundant star trackers. Coarse sun sensors are utilized as attitude reference devices during safe hold mode. Three two-axis gyros measure SC inertial rates to assist closed-loop slewing operations, providing full redundancy. A three-axis magnetometer senses the earth's magnetic field orientation, while six reaction wheels provide the necessary torque for slews, and manage and store momentum. Three redundantly-wound magnetic torquer bars unload excess momentum.

Electrical Power-Swift's power source is a pair of singleaxis gimbaled solar array wings. Together the pair of threepanel wings produces a total of 2,215 watts at EOL. Energy storage is provided by a single 80 amp-hour Individual

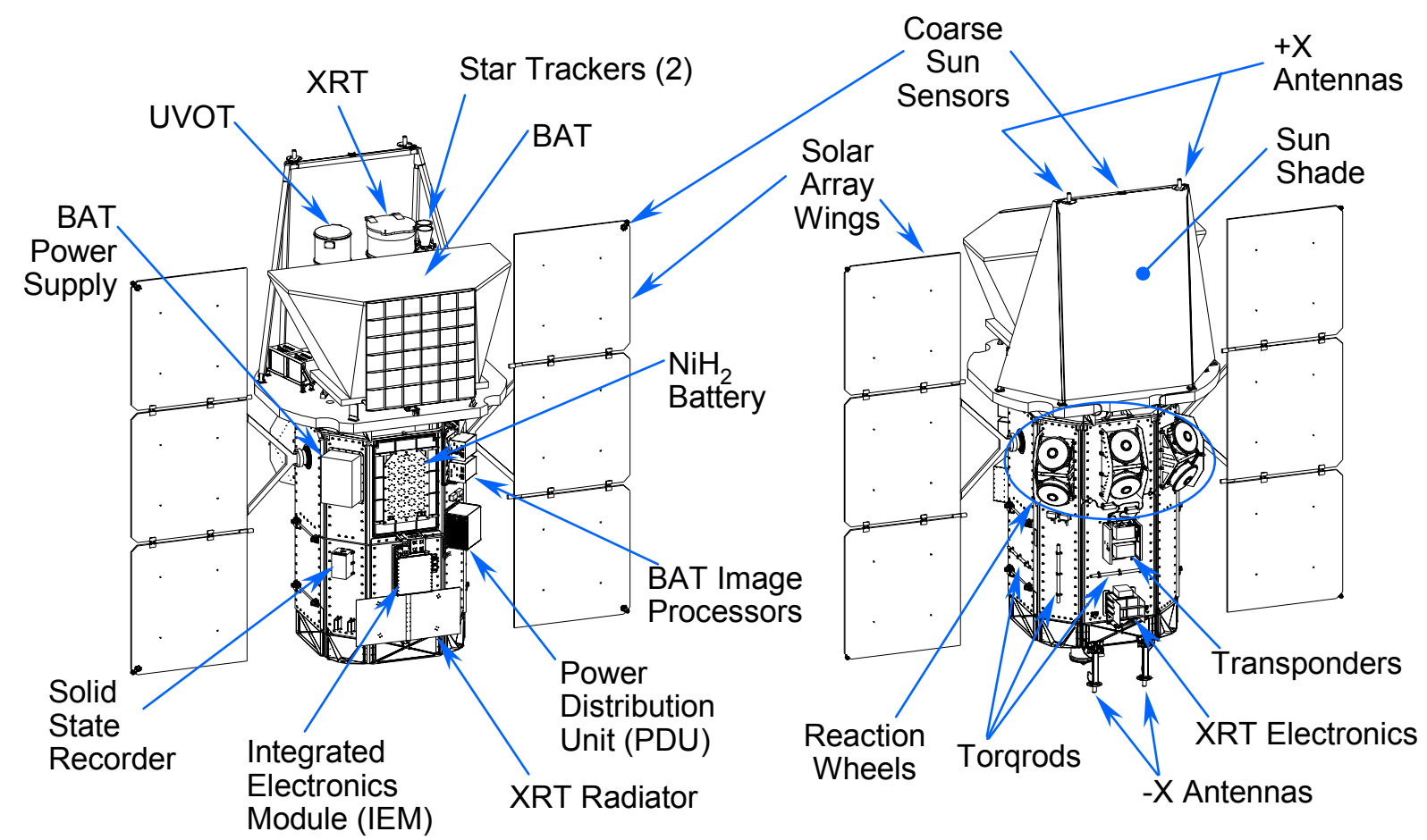

Figure 10 - Swift SC Configuration 


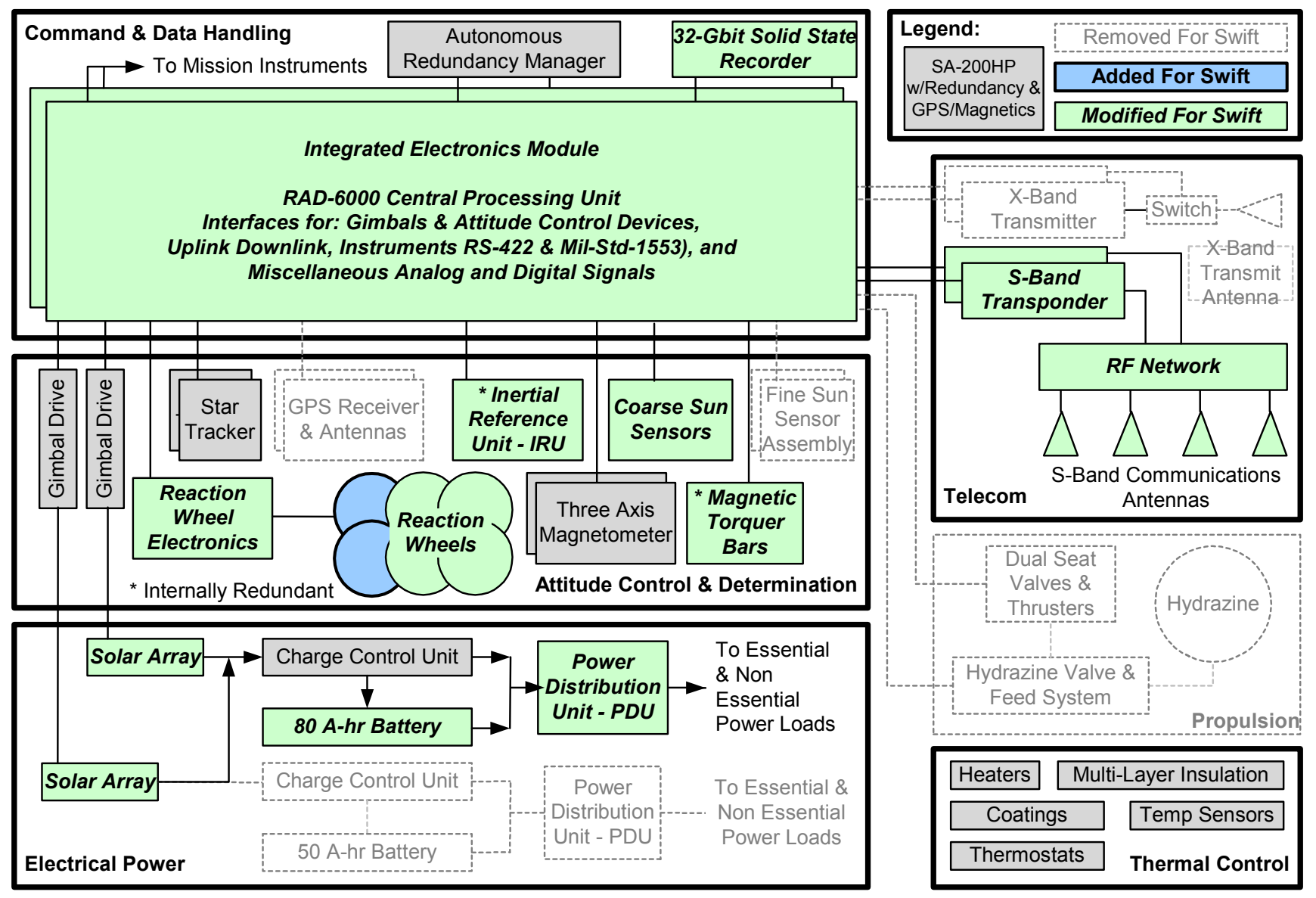

Figure 11 - Swift Functional Block Diagram Showing Revisions, Additions and Deletions to the SA-200HP Baseline SC Bus Design

Pressure Vessel (IPV) $\mathrm{NiH}_{2}$ battery regulated by an internally redundant CCU. An internally redundant PDU provides switching of non-essential power loads.

Telecom-Swift utilizes the S-band Ground Network (GN) communications for normal command, telemetry, and science data downlink. Commanding is at $2 \mathrm{kbps}$ and telemetry is at $128 \mathrm{kbps}$. Science data downlink is at 2.5 Mbps. A set of diplexers, couplers, transfer switches and high speed electronic switches matrix the redundant set of TDRSS compatible transponders to a set of four omnidirectional antennas, assuring 4-pi steradian coverage. TDRSS Multiple Access (MA) communications links are utilized for immediate ground notification of GRB alerts. To maximize Radio Frequency (RF) uplink power to TDRSS, the SC has a set of transmit antennas controlled by a highspeed electronic RF switch. Since the SC's ACS knows its location in orbit and its geometric relationship with TDRSS, the system is able to autonomously switch to the antenna that provides the best link geometry to TDRSS at all times. In the event of an anomaly or loss of attitude, the SC broadcasts a signal alternately through each of the two antennas for full hemispherical coverage. Emergency commands may also be sent through the TDRSS network to Swift.

Propulsion-Swift has no requirement for a propulsion subsystem, so the SA-200HP propulsion subsystem was not included.

Structures/Mechanisms and Thermal Control-As with the SA-200HP and Coriolis SC, the Swift structure is fabricated from aluminum stringers, longerons, and honeycomb panels. The star trackers are mounted to Swift's XRT instrument to minimize structural misalignment. The thermal control subsystem is cold-biased with radiators and MLI blankets to minimize fluctuations in temperature. Thermostatically controlled heaters are used to keep components at desired temperatures.

\section{SIMILARITIES AND DIFFERENCES BETWEEN CORIOLIS AND SWIFT}

This Section discusses many of the similarities and differences between the Swift and Coriolis missions in terms of mission functionality and requirements. Figure 13 contains a table, which quantifies the differences in terms of the resulting performance of the Coriolis and Swift missions. The table also allows side-by-side comparisons of the Swift 


\begin{tabular}{|c|c|c|}
\hline Subsys & Modification & Rationale \\
\hline \multirow[t]{2}{*}{ C\&DH } & $\begin{array}{l}\text { Changed SSR size from } 100 \text { Gbits to } 32 \\
\text { Gbits }\end{array}$ & $\begin{array}{l}\text { Swift mission data storage requirement can be satisfied } \\
\text { with smaller unit }\end{array}$ \\
\hline & Deleted propulsion interface & No propulsion system \\
\hline Structure & $\begin{array}{l}\text { Modify SA-200HP structure to } \\
\text { accommodate Swift instruments }\end{array}$ & Mission requirement \\
\hline \multirow[t]{4}{*}{ ADCS } & $\begin{array}{l}\text { Replaced high precision IRU with moderate } \\
\text { precision IRU }\end{array}$ & IRU selection dictated by mission requirements \\
\hline & Deleted GPS Receiver & Location accuracy not required for Swift mission \\
\hline & Deleted fine sun sensors & Not necessary for mission operation \\
\hline & $\begin{array}{l}\text { Increased reaction wheel quantity from } 4 \text { to } \\
6 \text { and modified reaction wheel and torquer } \\
\text { bar sizes }\end{array}$ & $\begin{array}{l}\text { Larger number of wheels and larger sizing of wheels and } \\
\text { torquer bars necessary to provide rapid slew capability; } \\
\text { manage and unload momentum }\end{array}$ \\
\hline Propulsion & $\begin{array}{l}\text { Deleted blow-down monopropellant } \\
\text { propulsion subsystem }\end{array}$ & Propulsion subsystem not required for Swift mission \\
\hline \multirow[t]{2}{*}{ Electrical Power } & $\begin{array}{l}\text { Delete dual axis gimbaled solar array; } \\
\text { replace with single axis gimbaled solar } \\
\text { arrays }\end{array}$ & $\begin{array}{l}\text { Dual axis gimbaled arrays are not necessary to meet } \\
\text { Swift power requirements }\end{array}$ \\
\hline & $\begin{array}{l}\text { Replace redundant } 50 \text { A-hr SPV batteries } \\
\text { with a single } 80 \text { A-hr IPV battery. }\end{array}$ & $\begin{array}{l}\text { Redundancy not required to meet Swift mission } \\
\text { reliability requirements; } 80 \text { A-hr battery required to meet } \\
\text { Swift eclipse requirements }\end{array}$ \\
\hline \multirow[t]{2}{*}{ Telecom } & Deleted X-band transmitter and antenna & $\begin{array}{l}\text { X-band deleted since Swift does not have a high } \\
\text { downlink data requirement }\end{array}$ \\
\hline & $\begin{array}{l}\text { Replace S-band transponder with TDRSS- } \\
\text { compatible unit }\end{array}$ & $\begin{array}{l}\text { Different transponder required for dual use (STDN and } \\
\text { TDRSS); mission requirement }\end{array}$ \\
\hline
\end{tabular}

Figure 12 - Subsystem Summary of Swift Mission Unique Changes to the SA-200HP SC Bus

and Coriolis missions with the SA-200HP core SC bus and the SA-200HP with the Full Redundancy option.

The Coriolis and Swift SC share a great deal of commonality in their basic system architecture and avionics. Both SC are block redundant and are designed for three to five year lifetimes. They utilize the same basic VME-based C\&DH subsystem and the same direct energy transfer EPS design using $\mathrm{NiH}_{2}$ batteries. Both employ stand-alone SSRs for science data storage. They feature onboard fault detection, safing, and load shedding. Both SC use S-band for command and telemetry communications. Both use star trackers and gyros for attitude determination, reaction wheels for attitude control, and magnetics (magnetometer and torquer bars) for momentum unloading. Both have coldbiased, passive thermal designs that rely on multi-layer thermal insulation, and thermostatically controlled heaters. Both missions were procured from the RSDO catalog under firm fixed price contracts using the basic SA-200HP core bus design, yet they have been adapted to perform quite different missions for different customers.

Swift is a NASA astronomy mission while Coriolis is an Air Force technology demonstration and earth sciences mission. The missions have significantly different orbits - Coriolis is placed in a high inclination, sun synchronous orbit while Swift is in a low inclination (non sun-synchronous) orbit. The two SC are launched with different LVs from different ranges - Coriolis is launched on a Titan II from the Western Test Range (WTR), while Swift is launched on a Delta II from the Eastern Test Range (ETR). Coriolis contains onboard propulsion for orbit raising and orbit adjustment maneuvers while Swift does not. Both SC utilize Spectrum Astro's aluminum "space frame" structural approach, but each SC is configured quite differently. Coriolis has the majority of its SC bus components mounted in the interior of the bus, which has been designed as an electromagneticallytight Faraday cage. Swift accommodates two very long telescopes going through the entire length of the bus with most of the SC bus components mounted on the exterior of the bus structure.

Coriolis maintains a constant nadir-looking attitude for performing earth science measurements and is designed as a pitch-momentum-bias system utilizing four reaction wheels. The Swift SC is configured as a ZMB system with large reaction wheels for rapid slewing to point virtually anywhere in the sky at astronomical targets. Because of the differing mission orbits and pointing requirements, Coriolis has a fixed panel solar array while Swift has an articulated array. Coriolis has onboard GPS receivers and a (ranging) transponder for precision timing and ranging, while Swift only requires North American Aerospace Defense Command (NORAD) data for ephemeris determination. Coriolis has high rate X-band science data downlink, whereas Swift uses S-band for science data transmission. Coriolis has real-time S-band tactical links to small ground terminals, while Swift has real-time TDRSS links. Coriolis, which is SGLS compatible, is being flown by the Air Force Research, Development, Test \& Evaluation (RDT\&E) Space Center (RSC) using the AFSCN Remote Tracking Sites (RTS) 


\begin{tabular}{|c|c|c|c|c|}
\hline Parameter & SA-200HP Core & \begin{tabular}{|c|} 
SA-200HP with \\
Full Redundancy \\
\& GPS/Magnetics \\
Options \\
\end{tabular} & Coriolis & Swift \\
\hline Target LV(s) & $\begin{array}{c}\text { Taurus, Athena I-II, } \\
\text { Titan II, Delta II } \\
\text { and Atlas }\end{array}$ & \begin{tabular}{|c|} 
Taurus, Athena I- \\
II, Titan II, Delta II \\
and Atlas
\end{tabular} & Titan II (WTR) & $\begin{array}{l}\text { Delta } 7420-10 \\
\text { (ETR) }\end{array}$ \\
\hline Mission Orbit \& Inclination & $\begin{array}{c}600 \times 600 \mathrm{~km} ; \\
28.5 \mathrm{deg} \\
\end{array}$ & $\begin{array}{c}600 \times 600 \mathrm{~km} ; \\
28.5 \mathrm{deg} \\
\end{array}$ & $\begin{array}{c}830 \text { x } 830 \mathrm{~km} ; \\
98.7 \mathrm{deg} \text { (sun sync) } \\
\end{array}$ & $\begin{array}{c}600 \times 600 \mathrm{~km} ; \\
22 \mathrm{deg}\end{array}$ \\
\hline Launch Date & N/A & $\mathrm{N} / \mathrm{A}$ & $15-$ Dec- 02 & 30-Sep-03 \\
\hline ACS Type & $\begin{array}{c}\text { 4-Wheel Zero } \\
\text { Momentum Bias } \\
\text { With Single Star } \\
\text { Tracker \& GPS } \\
\text { Receiver } \\
\end{array}$ & $\begin{array}{c}\text { 4-Wheel Zero } \\
\text { Momentum Bias } \\
\text { With Dual Star } \\
\text { Trackers \& GPS } \\
\text { Receivers } \\
\end{array}$ & $\begin{array}{c}\text { 4-Wheel Pitch } \\
\text { Momentum Bias } \\
\text { With Dual Star } \\
\text { Trackers \& GPS } \\
\text { Receiver } \\
\end{array}$ & $\begin{array}{c}\text { 6-Wheel Zero } \\
\text { Momentum Bias } \\
\text { With Dual Star } \\
\text { Trackers (No GPS } \\
\text { Receiver) } \\
\end{array}$ \\
\hline SC Bus Dry Mass (kg) & 354 & 374 & 354 & 613 \\
\hline Payload/Instrument Mass (kg) & 666 & 646 & 339 & 843 \\
\hline Observatory Dry Mass (kg) & 1020 & 1020 & 693 & 1456 \\
\hline Propellant Mass (kg) & 67 & 67 & 82 & 0 \\
\hline Observatory Wet Mass (kg) & 1087 & 1087 & 775 & 1456 \\
\hline Delta V Capability (m/s) & 137 & 137 & 228 & 0 \\
\hline Bus Power (W OAP) & 356 & 367 & 251 & 353 \\
\hline Payload Power (W OAP) & 650 & 639 & 421 & 695 \\
\hline Total Power (W OAP) & 1006 & 1006 & 672 & 1048 \\
\hline Solar Array Output (W EOL) & 2000 & 2000 & 1174 & 2215 \\
\hline Battery Type & $\mathrm{NiH}_{2} / \mathrm{SPV}$ & $\mathrm{NiH}_{2} / \mathrm{SPV}$ & $\mathrm{NiH}_{2} / \mathrm{SPV}$ & $\mathrm{NiH}_{2} / \mathrm{IPV}$ \\
\hline Battery Size & Two@50 Ahr & Two@ @ $50 \mathrm{Ahr}$ & One @ $50 \mathrm{Ahr}$ & One @ $80 \mathrm{Ahr}$ \\
\hline Uplink Rate (kbps) & 2 & 2 & 2 & $\begin{array}{c}2 \\
125 \text { bps TDRSS } \\
\end{array}$ \\
\hline Science Downlink Rate & $\begin{array}{c}80 \text { Mbps } \\
2.5 \text { Mbps backup }\end{array}$ & $\begin{array}{c}80 \text { Mbps } \\
2.5 \text { Mbps backup }\end{array}$ & $\begin{array}{c}25.6 \text { / } 51.2 \mathrm{Mbps} \\
256 \mathrm{kbps} \text { real-time }\end{array}$ & $\begin{array}{c}2.25 \mathrm{Mbps} \\
1 \mathrm{kbps} \text { TDRSS }\end{array}$ \\
\hline Telemetry Downlink Rate (kbps) & 128 & 128 & 128 & in $2.25 \mathrm{Mbps}$ link \\
\hline Data Storage (Gbits EOL) & 100 & 100 & 34 & 32 \\
\hline
\end{tabular}

Figure 13 - SC Performance Characteristics -

All Performance Numbers are Referenced to the Orbit and Inclination of Each Mission

located around the globe. Swift uses a STDN-compatible communications design and will be flown by a new, dedicated Mission Operations Center at Penn State University using an Italian Space Agency remote ground station at Malindi, Kenya.

In summary, the RSDO catalog approach offers a great deal of adaptability and flexibility in meeting unique mission requirements. While most SC components remain the same from mission to mission, they can be configured differently and augmented with additional components (or upgraded) as necessary to produce $\mathrm{SC}$ buses that can perform very different missions.

\section{APPLICATION OF GSFC'S RSDO CATALOG}

Perhaps the most common misconception of the RSDO catalog is for potential users to assume the core bus designs are static, i.e., they are fixed, build-to-print SC applicable to a specific range of orbit parameters. While the catalog describes a specific core bus configuration and provides system parameters for a given reference orbit (altitude and inclination), such data is offered only to illustrate bus capabilities. More often than not, the catalog serves only as a starting point, especially for science missions, and mission-unique modifications will be implemented to accommodate instrument layout and power/data interfaces, space-ground communications, and operations. Modest modifications to structure, solar array sizing, attitude control equipment, thermal control design, and data rates and formats are typical, as these items are strongly dependent on the instrument configuration. Tailoring them to the mission can result in significant cost and mass savings. Changes to the C\&DH handling, power, and communications subsystems are also possible. However, the trade between costs to modify and benefits to other performance 
parameters often indicates that retaining the core system design with some excess capability is the most effective approach. Most RSDO catalog SC buses represent heritage to a design from a specific mission that has been generalized to the catalog. In addition to the basic catalog SC bus, a SC vendor may have heritage subsystem and specialty interface designs from other flights, or there may be flight-proven commercially available components (e.g., attitude control sensors, transponders, transmitters, etc.) that can be substituted into the core with improved performance, low risk, and significant cost savings for a given mission.

In recognition of the widely varying mission requirements presented to RSDO, most SC vendors have a "family" of buses in the catalog that, although derived from discrete designs for specific missions, naturally extend into a continuum of overlapping mission scenarios. With such a broad SC base available from the catalog and the opportunity to implement mission-unique modifications, it is incumbent on the potential buyer to evaluate the core bus offerings based on potential for the mission application and to compare the capabilities of each vendor carefully.

Each catalog core bus includes assumptions on I\&T, launch operations, flight operations, handling and transportation, etc. which are captured in a Contract Data Requirements List (CDRL) submitted with the core description. These form the basis for the Not-to-Exceed (NTE) costs carried in the catalog. While any of the core bus features or program requirements can be tailored to mission requirements, the catalog assumptions apply unless otherwise stated in the delivery order. Buyers must be sure to review the core documents to ensure they are commensurate with their mission. In the event no delivery order changes are specified, the core terms and conditions apply. Subsequent changes are possible, but a contract modification is necessary.

\section{Conclusions}

Standard core bus designs and use of catalog procurement vehicles offer science and technology developers benefits in defining missions that are responsive to technical goals, while reducing costs and schedule associated with SC bus development and manufacture. The fight/qualification heritage of core buses minimizes risk by offering demonstrated performance and documented baseline designs. Moreover, the RSDO Rapid II catalog offers a streamlined procurement processes due to existing contractual vehicles and pre-defined terms and conditions.

Because of the uniqueness of science and technology missions, catalogs must allow for reasonable modification of core SC bus entries in order to accommodate mission-unique features. Correspondingly, buyers must recognize that SC catalogs reflect a vendor's capabilities. Although it is possible to procure a build-to print bus, in most likelihood mission specific modifications should be expected.
Consequently, the buyer should evaluate catalog buses on their potential, looking for a solid core capability to build on with the flexibility to be responsive to instrument accommodation. The Spectrum Astro Coriolis and Swift SC designs are excellent examples of how a single catalog core bus can be successfully adapted to a variety of scientific missions.

\section{REFERENCES}

[1] NASA Goddard Space Flight Center Rapid Spacecraft Development Office, Rapid II Catalog Website, http://rsdo.gsfc.nasa.gov/Rapidii/catalog2.cfm

[2] Marc D. Rayman, Philip Varghese, David H. Lehman, and Leslie L. Livesay, "Results from the Deep Space 1 Technology Validation Mission", 50th International Astronautical Congress, Amsterdam, The Netherlands, 4-8 October, 1999, IAA-99-IAA.11.2.01

[3] Jim Adams, RSDO Chief, "USAF Selects a Vendor for Coriolis Mission”, Rapid Spacecraft Development Office News, March 1999

http://rsdo.gsfc.nasa.gov/Newsletter/Archives/9903_rsdo.pdf

[4] WindSat Payload Description Website, http://www.pxi.com/windsat/descript.html

[5] Scott Greatorex/RSDO Mission Integration Manager, "Swift Selected as MIDEX Mission", Rapid Spacecraft

Development Office News, January 2000

http://rsdo.gsfc.nasa.gov/Newsletter/Archives/0001_rsdo.pdf

[6] Swift Mission Description Website, http://swift.gsfc.nasa.gov/scitech/mission/

[7] Marc D. Rayman and Philip Varghese, "The Deep Space 1 Extended Mission" Acta Astronautica 48, No. 5-12, pp. 693-705 (2001)

[8] Marc D. Rayman “The Deep Space 1 Extended Mission: Challenges in Preparing for an Encounter with Comet Borrelly", Acta Astronautica 51, No. 1-9, pp. 507-516 (2002)

[9] NASA-JPL Press Release Deep Space 1 Mission Status - "Deep Space 1 Flies By Comet Borrelly", September 22, 2001

http://www.jpl.nasa.gov/releases/2001/release_2001_189.html

[10] Spectrum Astro Coriolis Spacecraft Datasheet, http://www.spectrumastro.com/PDFs/Coriolis-Web.pdf

[11] Spectrum Astro Swift Spacecraft Datasheet, http://www.spectrumastro.com/PDFs/Swift-Web.pdf 


\section{BIOGRAPHIES}

Ed Belte is a Spacecraft Systems Engineer and Proposal/Program Manager. He has led development of several spacecraft proposal efforts and programs at TRW, Orbital Sciences and, currently, Spectrum Astro. He recently served as the study manager for the Geospace Electrodynamic Connections mission, a four

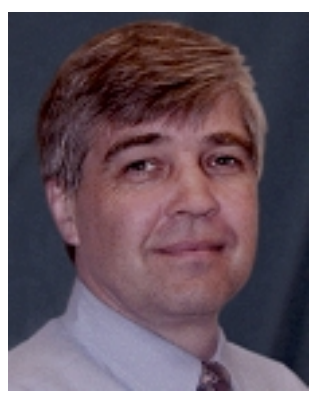
spacecraft constellation dipping into earth's IonosphereThermosphere (IT) to observe the magnetospheric energy transfer to the IT. Earlier in his career, he was the Mechanical Systems Manager for NASA's TDRSS flight 7 spacecraft and Program Manager for the Air Force's Space Test Experiment Platform-Mission 2. He holds a Bachelor of Engineering, Mechanical Engineering degree from The City College of New York and a Master of Science, Aerospace Engineering degree from Northrop University, Los Angeles, California.

Steve Thompson is a spacecraft and space mission Systems Engineer. He has developed and led numerous space systems architecture studies and their optimization at Spectrum Astro and TRW. He is currently leading Spectrum Astro's study efforts for the Magnetospheric Multi-Scale (MMS) mission. Earlier in his

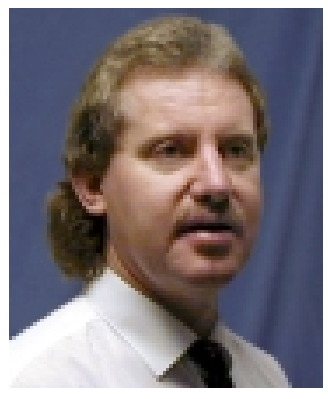
career, he also led conceptual spacecraft design studies for the Air Force's Space Test Experiment Platform-Missions 2, 3,4 and he led mission utility optimization studies for various national systems. He holds a Bachelor of Science, Aero/Astro Engineering degree from Ohio State University and a Master of Science, Aerospace Engineering degree from the University of Southern California.

\section{Dominick Conte is the Director of} Spectrum Astro's Herndon, Virginia field office. He also performs systems engineering and management of spacecraft conceptual designs for NASA and DoD missions. He led the development studies and provided the initial program management for Spectrum Astro's Swift Gamma

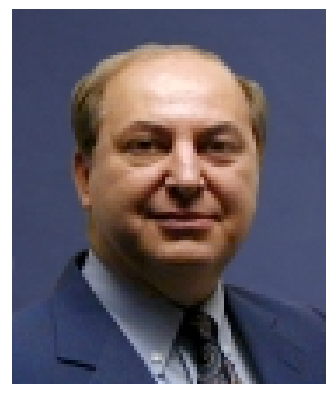
Ray Burst MIDEX spacecraft. Earlier in his career at TRW, he was Instrument Integrator, and later, Program Manager for the Air Force's Space Test Experiment Platform-Mission 1. He holds Bachelor and Master of Science degrees in Electrical Engineering from Syracuse University.
John Jordan is the Spectrum Astro Systems Engineering manager for the Swift Gamma Ray Burst mission. Previously, he was the lead Spectrum Astro systems engineer for the RHESSI Small Explorer (SMEX) spacecraft, and the New Millenium DS1 spacecraft. Before coming to Spectrum Astro, John was a Systems Engineer at

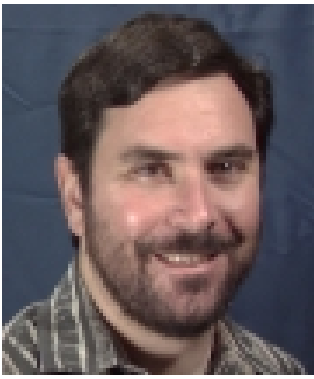
Hughes Space and Communications (now Boeing Space Systems), where he worked on several commercial communications satellite programs including DirecTV, Optus-A, Optus-B, and the HS-601 spacecraft bus development program. He holds a Bachelor of Science degree in Electrical Engineering from the Massachusetts Institute of Technology and a Master of Science degree in Electrical Engineering from the University of California at Los Angeles.

Al Lepore is the Spectrum Astro Program Manager for the Swift Spacecraft program. Prior to leading the Swift program, he held several managerial positions in the spacecraft thermal products line of business, both at Spectrum Astro and for Swales Aerospace. Earlier in his career while at General Electric (now Lockheed Martin), he

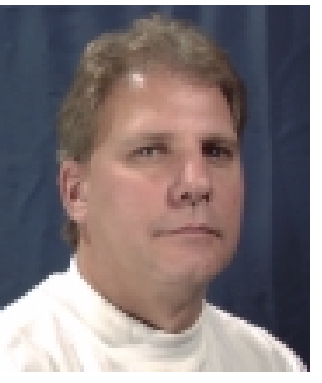
supported several spacecraft programs as a Thermal Subsystem Engineer. Al also holds two patents in the area of spacecraft thermal materials. He holds a Bachelor of Arts degree in Mathematics from Bloomsburg State College, Bloomsburg, Pennsylvania.

Kendall Nii is the Spectrum Astro Systems Engineer for the Coriolis spacecraft. Previously he performed systems engineering and mission operations development at Lockheed Martin Astronautics on the Mars Surveyor 98 and 2001 missions. Earlier in his career, while at Lawrence Livermore National Laboratories (LLNL) he

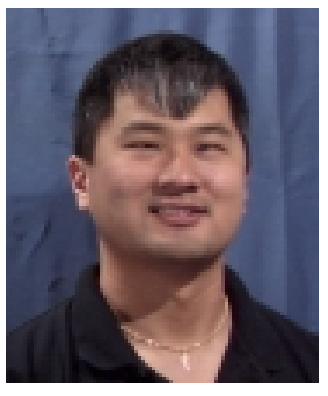
developed a solar cell test-bed for a long duration, high altitude, solar powered airplane called RAPTOR. Kendall holds a Bachelor of Science, Master of Science and PhD degrees in Aerospace Engineering, all from the University of Colorado at Boulder. 
David Olschansky is the Director of NASA, Civil and Technology Programs for Spectrum Astro. He is responsible for broadening Spectrum's business base in those areas and ensuring performance for the company's established hardware contracts, including the HESSI, Coriolis,

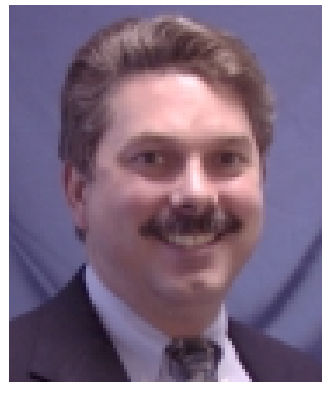

Swift, C/NOFS, and GLAST programs. Before coming to Spectrum Astro, David held engineering and management positions at Lockheed Martin on a broad range of space programs, including the Mars Odyssey, TIROS, DMSP, EOS TERRA, and Landsat 7, Earth Observing System (EOS) Common Spacecraft program, the electrical subsystems for the NASA Flight Telerobotic Servicer. He was also Chief Engineer for the NASA Magellan space vehicle, and the digital controller for the NASA Space Shuttle Main Engine (SSME). He holds a Bachelor of Science degree in Electrical Engineering from the University of Michigan in Ann Arbor.
John Sutila is the Spectrum Astro Coriolis Program Manager. He has spent the last 12 years in various capacities in the development of lightweight spacecraft, including six years at TRW. At Spectrum Astro, John supported the DS1 and RHESSI programs, as well as several successful space mission proposals. While much of his early career emphasis was on attitude determination and control systems design, John has also held subsystem, system engineering, payload integration, test, and on-orbit operations responsibilities, spanning six spacecraft. In 1994, he was the lead for the on-orbit recovery to operational status of the TAOS mission. He holds a Bachelor of Science degree in Aeronautical and Astronautical Engineering, and a Master of Science degree in Aeronautics and Astronautics, both from Purdue University. 